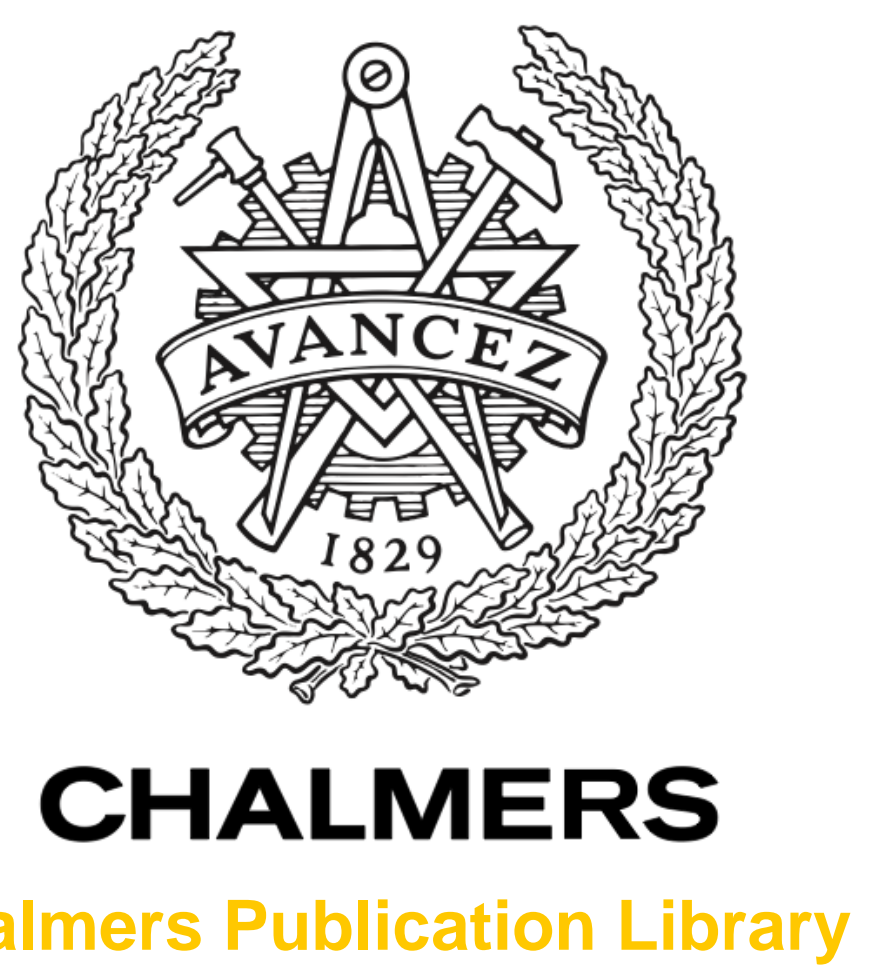

\title{
Accurate Beam Prediction Through Characteristic Basis Function Patterns for the MeerKAT/SKA Radio Telescope Antenna
}

This document has been downloaded from Chalmers Publication Library (CPL). It is the author's version of a work that was accepted for publication in:

IEEE Transactions on Antennas and Propagation (ISSN: 0018-926X)

Citation for the published paper:

Young, A. ; Maaskant, R. ; Ivashina, M. (2013) "Accurate Beam Prediction Through

Characteristic Basis Function Patterns for the MeerKAT/SKA Radio Telescope Antenna".

IEEE Transactions on Antennas and Propagation, vol. 61(5), pp. 2466-2473.

http://dx.doi.org/10.1109/TAP.2013.2239954

Downloaded from: http://publications.lib.chalmers.se/publication/180213

Notice: Changes introduced as a result of publishing processes such as copy-editing and formatting may not be reflected in this document. For a definitive version of this work, please refer to the published source. Please note that access to the published version might require a subscription. 


\title{
Accurate Beam Prediction Through Characteristic Basis Function Patterns for the MeerKAT/SKA Radio Telescope Antenna
}

\author{
A. Young, R. Maaskant, Member, IEEE, M. V. Ivashina, Member, IEEE, D. I. L. de Villiers, Member, IEEE, \\ and D. B. Davidson, Fellow, IEEE
}

\begin{abstract}
A novel beam expansion method is presented that requires employing only a few Characteristic Basis Function Patterns (CBFPs) for the accurate prediction of antenna beam patterns. The method is applied to a proposed design of the MeerKAT/SKA radio telescope, whose antenna geometry is subject to small deformations caused by mechanical or gravitational forces. The resulting deformed pattern, which is affected in a nonlinear fashion by these deformations is then sampled in a few directions only after which the interpolatory CBFPs accurately predict the entire beam shape (beam calibration). The procedure for generating a set of CBFPs - and determining their expansion coefficients using a few reference point sources in the sky - is explained, and the error of the final predicted pattern relative to the actual pattern is examined. The proposed method shows excellent beam prediction capabilities, which is an important step forward towards the development of efficient beam calibration methods for future imaging antenna systems.
\end{abstract}

Index Terms-reflector antennas, radio telescopes, characteristic basis function patterns, beam modeling, calibration.

\section{INTRODUCTION}

C ALIBRATION measurements on cosmic radio sources or geostationary satellites are often used to predict the far-field patterns of directive antennas, such as antennas for ground-based radio and (sub-) millimeter space telescopes. For these applications, it is very important to determine the antenna pattern down to the noise floor level in the measurements [1]-[3]. In practice, however, it is difficult to achieve this high accuracy due to atmospheric propagation (i.e. emission, absorption) and instrumental effects. The most important instrumental effects are: (i) the direction dependent signal-to-noise-ratio (SNR) for observing the reference source over the desired region of the measured beam, and; (ii) the

Manuscript received April xx, 20xx; revised January xx, 20xx. This work was supported in part by the South African Research Chairs Initiative of the Department of Science and Technology (DST) and National Research Foundation (NRF). Any opinion, findings and conclusions or recommendations expressed in this material are those of the authors and therefore the NRF and DST do not accept any liability with regard thereto. This work has been partly financed by the Netherlands Organisation for Scientific Research (NWO), the Swedish Agency for Innovation Systems VINNOVA, and the Swedish Research Council VR.

A. Young, D. I. L. de Villiers, and D. B. Davidson are with the Department of Electrical and Electronic Engineering, University of Stellenbosch, Stellenbosch, South Africa, e-mails: ayoung@sun.ac.za; ddv@sun.ac.za; davidson@sun.ac.za.

R. Maaskant and M. V. Ivashina are with the Signals and Systems Department of Chalmers University of Technology, Gothenburg, Sweden, emails: rob.maaskant@chalmers.se; marianna.ivashina@chalmers.se. system instability due to $e . g$. mechanical deformations of the antenna structure and/or variation of the receiver gain.

Conventionally used pattern calibration models used in radio astronomy applications are only accurate down to a few percent relative to the beam maximum [3]-[6], and reaching the required levels of accuracy presents a great challenge, especially given that the pattern models may need to be estimated repeatedly during the course of an observation in order to calibrate for the time-varying radiation characteristics of the antenna [7]. This limits the time practically available for performing the calibration measurements necessary for beam model estimation, thus precluding measurement on a directionby-direction basis.

Recently, a different class of pattern measurement techniques has been proposed that incorporates $a$ priori information about the antenna structure through employing physicsbased beam prediction models, thereby reducing the degrees of freedom for modeling the pattern significantly [8], [9]. Among these, a novel method has been proposed which requires employing only a few Characteristic Basis Function Patterns (CBFPs) for the accurate expansion of the antenna beam pattern [10]. The expansion coefficients were found by measuring the antenna array response on a few calibration sources only. In that specific case, it was shown that the perturbed array embedded element pattern due to impedance matching errors could be accurately predicted. This is an important step forward towards the development of efficient beam calibration methods for future imaging antenna systems, such as for the Square Kilometre Array, where fast and accurate calibration and imaging techniques are considered to be major research topics [11].

In this paper we propose to employ CBFPs for the first time for single-beam antenna systems. The objective is to accurately predict the beam pattern of a proposed design of the offset Gregorian MeerKAT/SKA Radio Telescope Antenna when this system is subject to geometrical deformations [12]. In Sec. II the method of employing CBFPs is described in a general manner, thereby demonstrating the close resemblance with the method of weighted residuals. The procedure for generating the CBFPs that can compensate for pattern errors caused by geometrical deformations is discussed in Sec. III. Finally, in Sec. IV, numerical results on the accuracy of the method are described. 


\section{Characteristic Basis Function Patterns}

Following the CBFP-method as presented in [13], but herein applied to a single beam antenna system, we aim to predict the actual reference beam pattern $\boldsymbol{F}^{\mathrm{ref}}(\Omega)$ - which may differ from the ideally expected pattern $\boldsymbol{F}^{\text {expt }}$ due to various errors in the system - through the model $\boldsymbol{F}^{\mathrm{mod}}(\Omega)$. To this end, the complex-valued vector function $\boldsymbol{F}^{\text {mod }}$ is expanded into a relatively small set of $N$ CBFPs $\left\{\boldsymbol{f}_{n}(\Omega)\right\}_{n=1}^{N}$, i.e.,

$$
\boldsymbol{F}^{\bmod }(\Omega)=\sum_{n=1}^{N} \alpha_{n} \boldsymbol{f}_{n}(\Omega)
$$

where $\left\{\alpha_{n}\right\}$ is the set of $N$ unknown CBFP expansion coefficients. Next, and analogously to the method of weighted residuals, the expansion coefficient vector $\boldsymbol{\alpha}=\left[\alpha_{1}, \alpha_{2}, \ldots, \alpha_{N}\right]^{T}$, where $T$ denotes transpose, is chosen such as to minimize the beam error function $\boldsymbol{\epsilon}(\Omega)=\boldsymbol{F}^{\mathrm{ref}}(\Omega)-\boldsymbol{F}^{\mathrm{mod}}(\Omega)$ over a desired angular region. This can be done by weighting $\epsilon$ to zero through the - yet to be chosen $-M$ testing functions $\left\{\boldsymbol{\Lambda}_{m}(\Omega)\right\}_{m=1}^{M}$, where $M \geq N$. Hence,

$$
\iint_{4 \pi} \boldsymbol{\Lambda}_{m} \cdot\left(\boldsymbol{F}^{\mathrm{ref}}-\boldsymbol{F}^{\mathrm{mod}}\right) \mathrm{d} \Omega=0
$$

where the symmetric product is used for testing. Substitution of (1) in (2) leads to the matrix equation

$$
\sum_{n=1}^{N}\left[\iint_{4 \pi} \boldsymbol{\Lambda}_{m} \cdot \boldsymbol{f}_{n} \mathrm{~d} \Omega\right] \alpha_{n}=\iint_{4 \pi} \boldsymbol{\Lambda}_{m} \cdot \boldsymbol{F}^{\mathrm{ref}} \mathrm{d} \Omega
$$

for $m=1,2, \ldots, M$. Henceforth, we will assume that the beam pattern is tested at $M$ discrete directions, i.e., is measured successively using $M$ known reference point sources in the sky, so that $\boldsymbol{\Lambda}_{m}(\Omega)=\boldsymbol{E}_{m}^{\mathrm{i}} \delta\left(\Omega-\Omega_{m}\right)$, where $\boldsymbol{E}_{m}^{\mathrm{i}}$ is the $E$-field polarization vector radiated by the $m$ th reference far-field source in the direction of the receiving antenna. The selection of Dirac distribution functions for testing the pattern (collocation method) is a logical choice since several relatively strong far-field point sources ${ }^{1}$ are readily available as natural calibrator sources [14]. Accordingly, Eq. (3) reduces to the matrix equation

$$
\mathbf{A} \alpha=\mathbf{V},
$$

where

$$
\mathrm{A}_{m n}=\boldsymbol{E}_{m}^{\mathrm{i}} \cdot \boldsymbol{f}_{n}\left(\Omega_{m}\right), \quad \mathrm{V}_{m}=\boldsymbol{E}_{m}^{\mathrm{i}} \cdot \boldsymbol{F}^{\mathrm{ref}}\left(\Omega_{m}\right) .
$$

One can solve for $\boldsymbol{\alpha}$ through the Moore-Penrose pseudoinverse, $\boldsymbol{\alpha}=\left(\mathbf{A}^{H} \mathbf{A}\right)^{-1} \mathbf{A}^{H} \mathbf{V}$, where the superscript $H$ denotes the conjugate transpose. Note that the choice of testing through a symmetric product in (2) is in correspondence with the reaction concept in electromagnetics, so that the element $\mathrm{V}_{m}$ in (5) is proportional to the measured antenna output voltage for the $m$ th source.

\footnotetext{
${ }^{1}$ Note that (3) allows us to measure (or test) on sky reference sources that are spatially distributed as well.
}

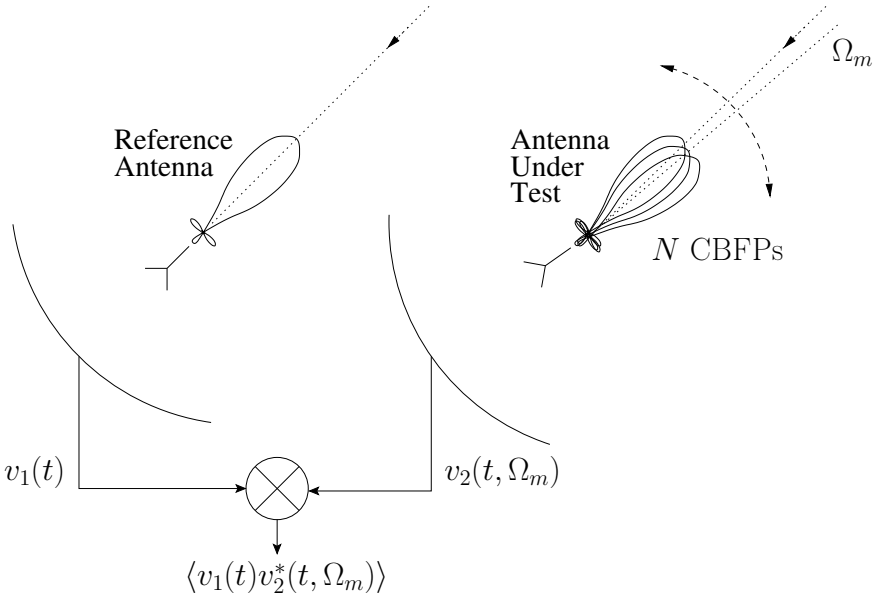

Fig. 1. Rotatable test antenna measuring a reference power point source in the $M$ different directions $\left\{\Omega_{m}\right\}_{m=1}^{M}$. The complex-valued receive voltages are obtained by correlating the respective output powers with a reference antenna pointing at the same source. Afterwards, the $N$ CBFP expansion coefficients are determined for modeling the unknown beam pattern of the antenna under test.

\section{A. Incoherent Power Point Sources}

In most practical cases the reference sources in the sky are natural incoherent power point sources. In that case, one instead will measure the real-valued time-averaged antenna output powers $p_{m}=\left\langle\left|\mathrm{V}_{m}(t)\right|^{2}\right\rangle$, for $m=1, \ldots, M$. Accordingly, from (4),

$$
\mathbf{p}=\operatorname{diag}\left(\left\langle\mathbf{V}(t) \mathbf{V}^{H}(t)\right\rangle\right)=\operatorname{diag}\left(\mathbf{A} \boldsymbol{\alpha} \boldsymbol{\alpha}^{H} \mathbf{A}^{H}\right)
$$

where $\operatorname{diag}(\mathbf{X})$ takes the diagonal of the square matrix $\mathbf{X}$ and places these elements in a column vector. To the authors' best knowledge, a closed-form solution to $\alpha$ in (6), given $\mathbf{p}$, does not exist, so that one has to resort to non-linear equation solvers [10]. Furthermore, since $\boldsymbol{\alpha} \boldsymbol{\alpha}^{H}=\boldsymbol{\alpha} \mathbf{Q} \mathbf{Q}^{H} \boldsymbol{\alpha}^{H}$, where $\mathbf{Q}$ is a unitary matrix (for example a diagonal matrix with arbitrary phase factors on its diagonal), the solution to $\boldsymbol{\alpha}$ suffers from a unitary matrix (or phase) ambiguity and is therefore not unique. Similarly, if in addition the reference sources are unpolarized, one also has to deal with a polarization ambiguity in the modeled pattern.

Alternatively, and rather than using a single antenna, one can directly measure the complex-valued receive voltage vector $\mathbf{V}$ in (4) by correlating the measured antenna output power with that of a reference antenna as shown in Fig. 1 ( $c f$. also [14], [15]).

After correlating the output signals, the measured $2 \times 2$ rank-one covariance matrix $\mathbf{R}\left(\Omega_{m}\right)$ is obtained as

$$
\mathbf{R}\left(\Omega_{m}\right)=\left[\begin{array}{cc}
\left\langle\left|v_{1}(t)\right|^{2}\right\rangle & \left\langle v_{1}(t) v_{2}^{*}\left(t, \Omega_{m}\right)\right\rangle \\
\left\langle v_{2}\left(t, \Omega_{m}\right) v_{1}^{*}(t)\right\rangle & \left\langle\left|v_{2}\left(t, \Omega_{m}\right)\right|^{2}\right\rangle
\end{array}\right]
$$

whose dominant eigenvector is composed of the complex voltage phasors $\left[V_{1} ; V_{2}\left(\Omega_{m}\right)\right]$, and has the eigenvalue $\lambda_{\max }=$ $\left|V_{1}\right|^{2}+\left|V_{2}\right|^{2}$. Hence, the element $V_{m}$ in (5) can be computed as $\bigvee_{m}=V_{2}\left(\Omega_{m}\right) / V_{1}$, where we normalized to $V_{1}$ in order to compensate for possible phase and amplitude variations of the reference source signal. 


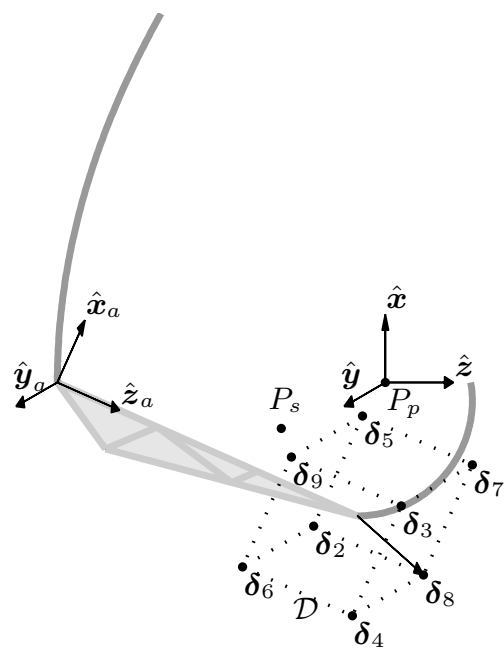

Fig. 2. Geometry of the proposed offset Gregorian MeerKAT system with the domain space of subreflector position errors $\mathcal{D}$ shown on an exaggerated scale for clarity. The vector $\boldsymbol{\delta}$ extends from the center of $\mathcal{D}$ which is located at the base of the subreflector (ideal position). The indicated positions $\boldsymbol{\delta}_{2}, \ldots, \boldsymbol{\delta}_{9}$ were used to construct the secondary CBFPs for set $S_{1}$. The position error of the feed (relative to the ideal location $P_{s}$ ) is proportional to that of the subreflector. $P_{p}$ indicates the primary focus point

\section{CBFP-Method Applied to MeERKAT}

\section{A. CBFP Generation}

The next step is to select a representative set of CBFPs, whose powerful property is that relatively few of them are required for the accurate modeling of the beams. We distinguish between primary and secondary CBFPs: (i) the primary CBFP, $f_{1}$, is a simulated or measured pattern corresponding to the ideally expected beam pattern, $\boldsymbol{F}^{\text {expt }}$, in the absence of system errors. This physics-based pattern alone is assumed to be already very close to the actual (slightly perturbed) reference pattern $\boldsymbol{F}^{\text {ref }}$; (ii) the secondary higher-order CBFPs, $\left\{\boldsymbol{f}_{n}\right\}_{n=2}^{N}$, need to be able to compensate for expected beam errors to be able to accurately predict $\boldsymbol{F}^{\text {ref }}$. The secondary CBFPs should therefore span the space of beam patterns that are to be expected when the antenna system is subject to certain types of errors. That is, the set of secondary CBFPs correspond to the radiation patterns of a set of possible systems that are representative of the various expected errors. This set of pattern basis functions may be determined through simulation (depending on how accurately the actual system with or without errors - can be modeled), or measurement (depending on how well representative error conditions can be enforced on the actual system), or a combination of both.

As an example, the set of CBFPs used to model antenna beams for a reflector system under varying thermal loading conditions may consist of a simulated pattern of the ideal antenna geometry (primary CBFP), and a number of measured patterns obtained at different times of the day (secondary CBFPs) [5, cf. Fig. 19]. If other types of geometrical errors are to be modeled as well (e.g. positioning error of the feed and/or subreflector, ground roughness, etc.), the set of secondary CBFPs needs to be augmented with basis function patterns obtained for the corresponding erroneous systems. Since the relation between geometrical deformations and radiation pat-
TABLE I

ESTIMATED TOLERANCES ON SUBREFLECTOR POSITION IN UNIT WAVELENGTHS [12].

\begin{tabular}{|l|c|c|}
\hline Direction & $580 \mathrm{MHz}$ & $1.75 \mathrm{GHz}$ \\
\hline$x_{a}$ & \pm 0.0193 & \pm 0.0583 \\
\hline$y_{a}$ & \pm 0.0097 & \pm 0.0292 \\
\hline$z_{a}$ & \pm 0.0387 & \pm 0.1167 \\
\hline
\end{tabular}

tern shape is generally non-linear, CBFPs obtained from system configurations containing multiple anticipated errors may also be required. However, depending on the magnitude of the expected errors (which are generally relatively small) the addition of more basis functions may result in a certain degree of redundancy, and an accurate pattern model may be achieved without using all the generated CBFPs. At any rate, a larger number of CBFPs may therefore be required to address the problem of modeling non-linear beam pattern variations for the geometrical errors discussed here, as opposed to e.g. beamformer weight errors which constitute a linear problem [10]. As an illustrative example of the proposed method, herein we will consider the case where the beam errors are a result of geometrical deformations resulting in displacement of the feed and subreflector only.

Consider the offset Gregorian candidate design for the MeerKAT antenna in Fig. 2 [16]. The estimated tolerances on the subreflector position are presented in Table I (for two different frequencies of operation) and are defined in the coordinates $\left(x_{a}, y_{a}, z_{a}\right)$ shown at the base of the support arm on which the feed and subreflector are mounted. The gain pattern variations caused by these geometrical variations have been examined in [12]. The geometrical position error of the feed and subreflector are proportionally affected since these components are supported by the same structure. Hence, we will define the subreflector position error as $\boldsymbol{\delta}=\left[\delta_{x}, \delta_{y}, \delta_{z}\right]^{T}$, from which the feed position error is determined as the ratio of the position of the feed along the support arm to that of the subreflector. Accordingly, the three-dimensional Euclidean space $\mathcal{D}$ of position errors is defined, whose domain is bounded by the values listed in Table I. Upon taking $K$ samples within $\mathcal{D}$, the set of geometrical error vectors $\left\{\boldsymbol{\delta}_{k}\right\}_{k=1}^{K}$ is obtained ${ }^{2}$. With the finite set of error vectors thus defined, the generation of the corresponding set of CBFPs can proceed.

The primary CBFP is chosen as the simulated far field pattern of the error-free antenna geometry, i.e. $\boldsymbol{f}_{1}=\boldsymbol{F}^{\operatorname{expt}}\left(\boldsymbol{\delta}_{1}=\right.$ 0). Similarly the set of secondary CBFPs is obtained through simulation and by setting $\boldsymbol{f}_{k}=\boldsymbol{F}^{\text {expt }}\left(\boldsymbol{\delta}_{k}\right)$ for $k=2, \ldots, K$. Using the so-generated initial set of $K$ CBFPs (primaries + secondaries), each of which is simulated at a total of $N_{\Omega}$ far field directions, a $2 N_{\Omega} \times K$ matrix $\mathbf{F}$ can be formed ( 2 far field components),

$$
\boldsymbol{F}=\left[\begin{array}{ccc}
\boldsymbol{F}^{\operatorname{expt}}\left(\Omega_{1}^{\mathrm{s}}, \boldsymbol{\delta}_{1}\right) & \cdots & \boldsymbol{F}^{\operatorname{expt}}\left(\Omega_{1}^{\mathrm{s}}, \boldsymbol{\delta}_{K}\right) \\
\vdots & \ddots & \vdots \\
\boldsymbol{F}^{\operatorname{expt}}\left(\Omega_{N_{\Omega}}^{\mathrm{s}}, \boldsymbol{\delta}_{1}\right) & \cdots & \boldsymbol{F}^{\operatorname{expt}}\left(\Omega_{N_{\Omega}}^{\mathrm{s}}, \boldsymbol{\delta}_{K}\right)
\end{array}\right],
$$

\footnotetext{
${ }^{2}$ The probablity density function of the displacement error can be used to obtain a non-uniform sampling grid.
} 
where $\Omega^{\mathrm{s}}$ denotes a sampled (simulated) far-field direction. A linear dependency between the angular sampled version of the CBFPs (i.e. columns of $\mathbf{F}$ ) may exist, which renders the matrix $\mathbf{F}$ rank-deficient. The CBFPs can be orthonormalized and redundant ones be eliminated through the application of the Singular Value Decomposition (SVD), as is done in the Characteristic Basis Function Method (CBFM) [17]. In the present context, we then retain only the first $N$ left-singular vectors of $\mathbf{F}$ as the reduced set of CBFPs, which are the ones corresponding to singular values whose magnitudes are above a certain specified threshold relative to the largest singular value. Furthermore, it is pointed out that several rows of $\mathbf{F}$ can be discarded during the SVD process if the objective of the CBFPs is to model the beam pattern only over a limited angular region. Generally, the smaller the angular region, the lesser the number of retained CBFPs. Afterwards, and if desired, the corresponding CBFP beam functions outside the limited angular support can be recovered through using the first $N$ right-singular vectors as expansion coefficient vectors for the initial set of CBFPs. This permits modeling the beam over the entire angular support, while the accuracy is highest within the limited angular region.

When measured (as opposed to simulated) CBFPs are employed, e.g. by manually introducing displacement errors and measuring the corresponding pattern functions, it becomes practically intractable to generate a large initial set of CBFPs. Therefore, we also consider the case of generating only $K=9$ initial simulated CBFPs, that is, at the center (primary CBFP) and corners (secondary CBFPs) of the cuboidal-shaped subdomain $\mathcal{D}$ ( $c f$. Fig. 2).

Hence, the following sets of CBFPs will be considered: (i) set $S_{1}$, composed of $K=9$ CBFPs (no SVD); (ii) set $S_{2}$, composed of $K=9$ CBFPs (after SVD on $S_{1}$ ); (iii) set $S_{3}$, composed of $K=351$ CBFPs (after SVD), which is obtained through regularly sampling the error subdomain $\mathcal{D}$. The SVD will only be used to orthonormalize the CBFPs, i.e. no threshold on the singular values is applied to reduce each set of CBFPs, so that $K=N$; instead, the model accuracy is examined as a function of the number of admitted CBFPs in decreasing order of importance (for $S_{2}$ and $S_{3}$ this is based on the magnitude of the singular values, while for $S_{1}$ the ordering is as indicated in Fig. 2).

\section{B. Selection of Testing Points}

The numerical solution accuracy of the expansion coefficient vector $\boldsymbol{\alpha}$ in (1), and therefore $\boldsymbol{F}^{\text {mod }}$, depends on the matrix condition number $\kappa$ of $\mathbf{A}$ in (4). In turn, $\kappa(\mathbf{A})$ depends on the total number and positions of the testing points. In the following, we will choose the total number of testing points $M$ equal to the total number of employed CBFPs $N$, and let the first testing point $\Omega_{1}$ correspond to the on-axis direction $\theta=0$. For each additional CBFP included in the model, the corresponding $\Omega_{n}$ to be selected next is chosen such as to increase $\kappa(\mathbf{A})$ by the least amount. Furthermore, the points are limited to a region $\theta<\theta_{\max }$ around the expected beam maximum where the antenna sensitivity is expected to attain high values as well. Note that the procedure for generating the

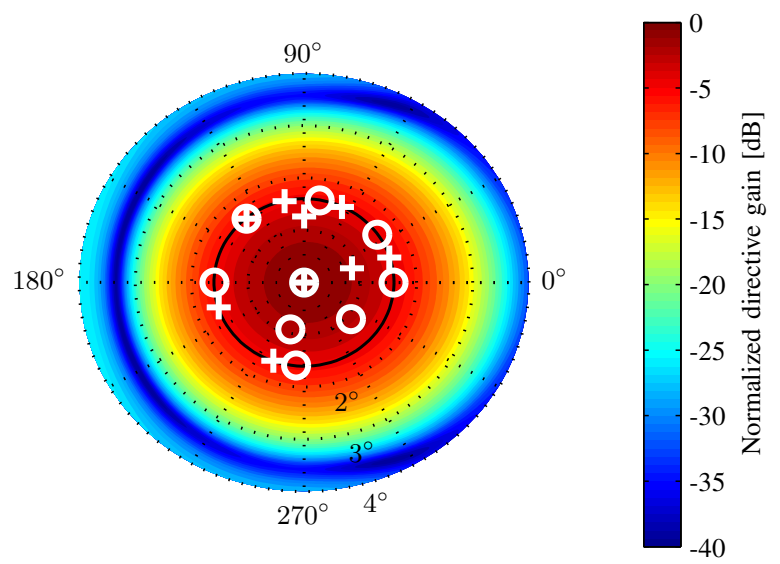

Fig. 3. Testing points for $S_{1}$ ('+') and $S_{2}$ ('o') shown on the ideally expected normalized gain pattern at $580 \mathrm{MHz}$. The contour $\theta=\theta_{\max }$ is indicated as a solid black line.

sets $\left\{\boldsymbol{f}_{n}\right\}$ and $\left\{\Omega_{n}\right\}$ needs to be performed only once for the construction of $\mathbf{A}$ in (4), whereas the forcing vector $\mathbf{V}$ needs to be updated at regular time intervals to calibrate the beam during operation.

Fig. 3 shows the resulting testing points for $S_{1}$ and $S_{2}$ at $580 \mathrm{MHz}$, where the value of $\theta_{\max }=1.6^{\circ}$ was selected so that all testing points are approximately within the $5 \mathrm{~dB}$ beam of the ideally expected pattern. As can be seen, most of the testing points were found to reside close to or on the boundary $\theta=\theta_{\max }$. At $1.75 \mathrm{GHz}$ a value of $\theta_{\max }=0.5^{\circ}$ was chosen (contour plot not shown).

\section{Numerical Results}

Fifty simulated reference patterns were analyzed by randomly $^{3}$ selecting position errors $\delta$ of the subreflector (and correspondingly of the feed) within $\mathcal{D}$. Accordingly, the $i$ th reference pattern, $\boldsymbol{F}_{i}^{\text {ref }}$, for $i \in\{1,2, \ldots, 50\}$, is predicted through the modeled pattern $\boldsymbol{F}_{i, j, N}^{\text {mod }}$ by employing $N$ CBFPs, where $N \in\{1,2, \ldots, 9\}$, and for the CBFP sets $S_{j}$, where $j \in\{1,2,3\}$. As a result, each of the 50 reference patterns was modeled in 27 different ways, which enabled us to perform a detailed comparative analysis. Afterwards, the pertaining pattern error function $\boldsymbol{\epsilon}_{i, j, N}$ was calculated for each of the cases and then normalized to the maximum of the corresponding reference pattern. Two error metrics were considered: the first error metric $\epsilon_{j, N}^{\mathrm{avg}}$ was chosen as the average of $\left|\epsilon_{i, j, N}\right|$ over the angular region where the normalized $\boldsymbol{F}_{i}^{\text {ref }}$ is above the $-10 \mathrm{~dB}$ level, and averaged over all $i$; the second error metric $\epsilon_{j, N}^{\max }$ was chosen as the maximum of $\left|\boldsymbol{\epsilon}_{i, j, N}\right|$ over the angular region where the normalized $\boldsymbol{F}_{i}^{\text {ref }}$ is above the $-30 \mathrm{~dB}$ level, and the worst case selected from all $i$. The generation of the CBFPs as well as the error calculations were performed separately at the two frequency points $580 \mathrm{MHz}$ and $1.75 \mathrm{GHz}$.

Fig. 4(a) shows the average error metric $\epsilon_{j, N}^{\mathrm{avg}}$ as a function of $N$ for the various sets of CBFPs at $580 \mathrm{MHz}$. The average error between the actual reference patterns and the

\footnotetext{
${ }^{3}$ A uniform probability distribution was assumed for $\delta$ within $\mathcal{D}$, which represents a worst-case scenario for the actual performance of the antenna in practice.
} 


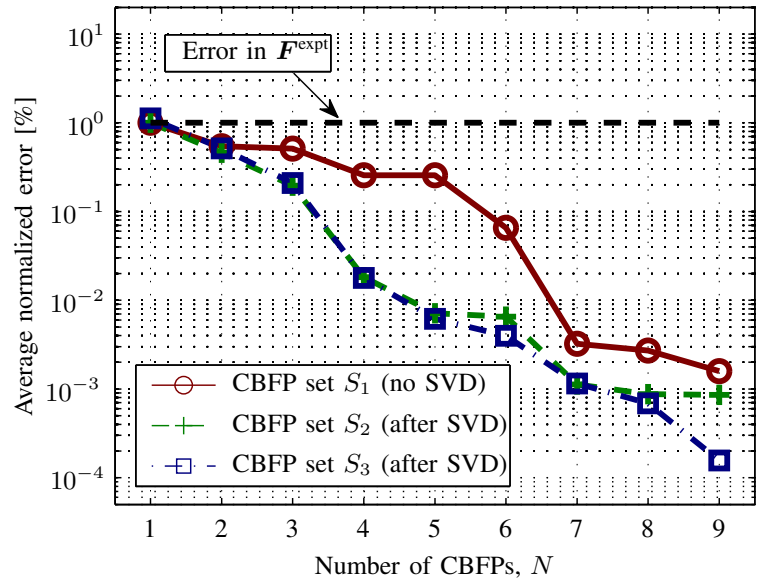

(a) $580 \mathrm{MHz}$

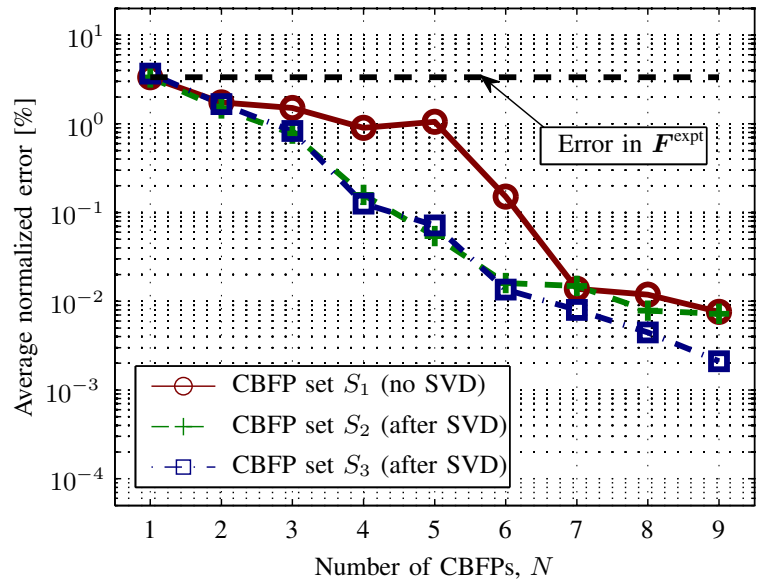

(b) $1.75 \mathrm{GHz}$

Fig. 4. Average error in the model pattern $\boldsymbol{F}^{\text {mod }}$ using different numbers of employed CBFPs $N$. The average error for all geometrical deformations is used and a comparison is shown between CBFPs from $S_{1}$ (without SVD), $S_{2}$ ( $S_{1}$ after SVD), and $S_{3}$ (after SVD).

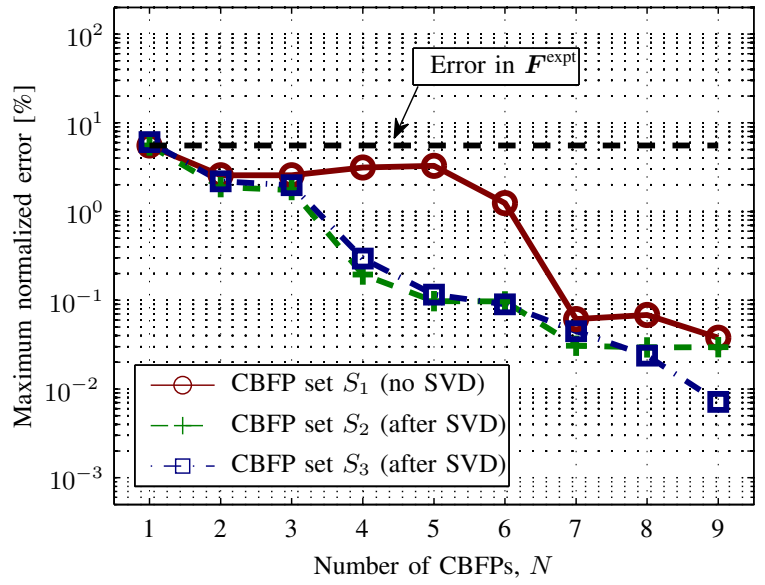

(a) $580 \mathrm{MHz}$

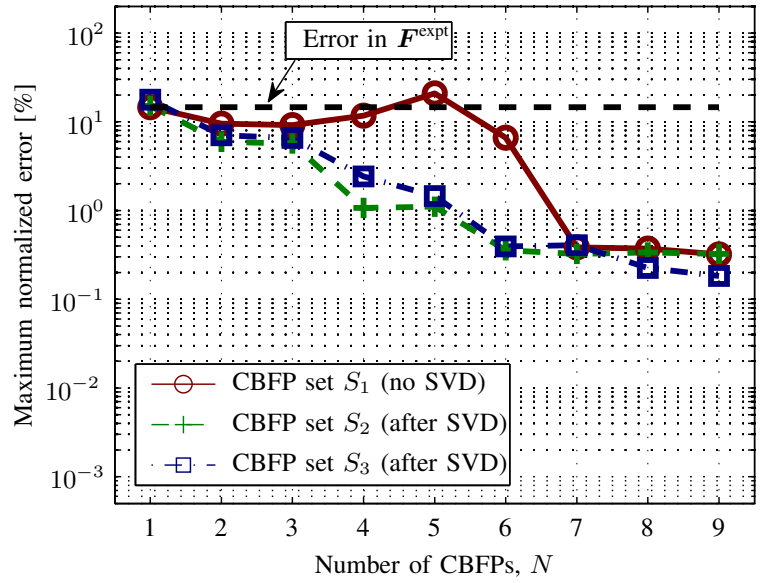

(b) $1.75 \mathrm{GHz}$

Fig. 5. Worst case maximum error in the model pattern $\boldsymbol{F}^{\text {mod }}$ obtained using different numbers of employed CBFPs $N$. Comparison is shown between CBFPs from $S_{1}$ (without SVD), $S_{2}\left(S_{1}\right.$ after SVD), and $S_{3}$ (after SVD).

corresponding ideally expected patterns (when $\boldsymbol{\delta}=\mathbf{0}$ ) is also shown in the figure by the black dashed line. Not surprisingly, a single CBFP from $S_{1}$ results in an error $(\approx 1 \%)$ which is exactly equal to that for the ideal pattern since the primary CBFP was chosen to be equal to $\boldsymbol{F}^{\operatorname{expt}}(\boldsymbol{\delta}=\mathbf{0})$. Similarly, the primary CBFPs for the sets $S_{2}$ and $S_{3}$ (obtained after the SVD) closely resemble $\boldsymbol{F}^{\text {expt }}$ and, consequently, the error for a single CBFP from these sets is at the same level as for $S_{1}$ and $\boldsymbol{F}^{\text {expt }}$. Upon increasing $N$, the error in the modeled pattern decreases relatively slowly when employing CBFPs from $S_{1}$, as opposed to the CBFPs from $S_{2}$, whose error reduces much more rapidly (even for small $N$ ) owing to the orthonormalization of the CBFPs over the angular region near the beam maximum. Ultimately, for $N=9$, the beam approximation errors for the sets $S_{1}$ and $S_{2}$ are the same, since both sets span the same space of beam patterns. However, employing CBFPs from set $S_{3}$, and for $N=9$ the error can be reduced further by about an order of magnitude. The method is shown to model the variation in the beam patterns with high accuracy, i.e., even with CBFPs from $S_{2}$ alone, the average error can be reduced to a level of about $10^{-3} \%(-100 \mathrm{~dB})$.

The average error computed at $1.75 \mathrm{GHz}$ is shown in Fig. 4(b) and the results are similar to that for the lower frequency. However, due to the larger electrical distance of the mechanical displacements at the higher frequency, the shape of the reference patterns deteriorate, generally resulting in a larger error between the reference and the modeled patterns (as well as between the reference and the ideally expected patterns). Also, the condition number $\kappa(\mathbf{A})$ is smaller at the higher frequency for the same set of CBFPs, which is a direct consequence of the increased effect of the geometrical errors on the beam patterns at the higher frequency. However, although the matrix condition number improved, more CBFPs may need to be employed to achieve the same accuracy at higher frequencies. Upon employing all nine CBFPs from either $S_{1}$ or $S_{2}$, the error can be reduced to as little as $10^{-2} \%$, and even further by a factor four when employing the same number of CBFPs from $S_{3}$. 


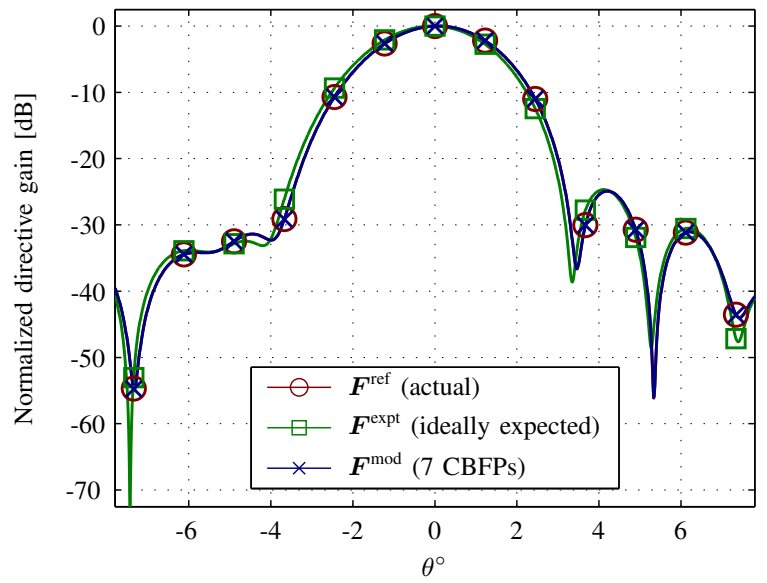

(a) Beam pattern @580 $\mathrm{MHz}\left(\phi=170.0^{\circ}\right)$

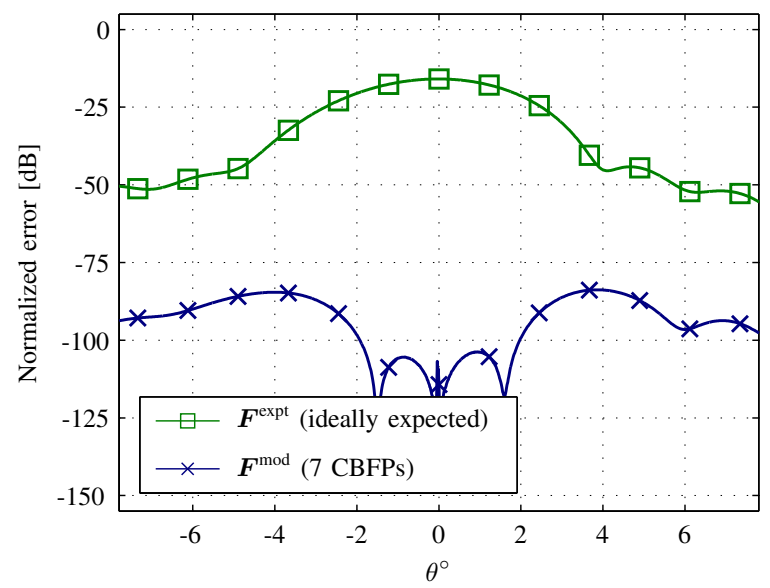

(c) Error function @ $580 \mathrm{MHz}\left(\phi=170.0^{\circ}\right)$

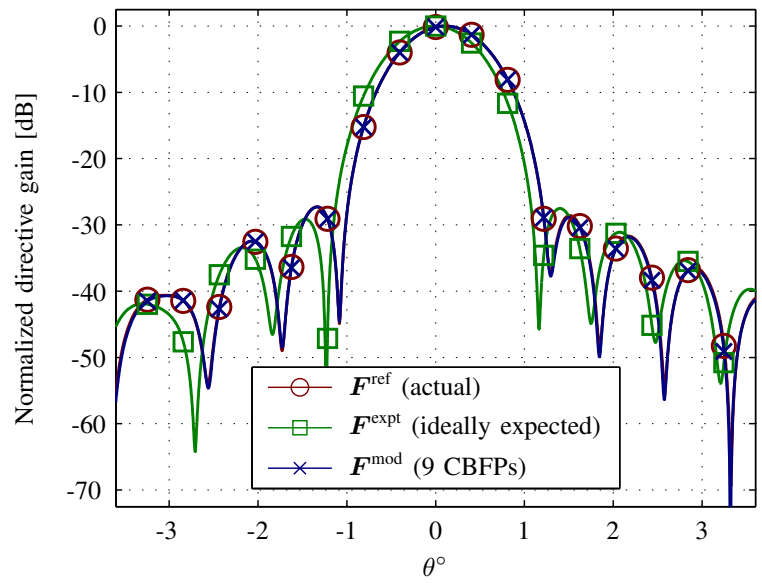

(b) Beam pattern @ $1.75 \mathrm{GHz}\left(\phi=157.5^{\circ}\right)$

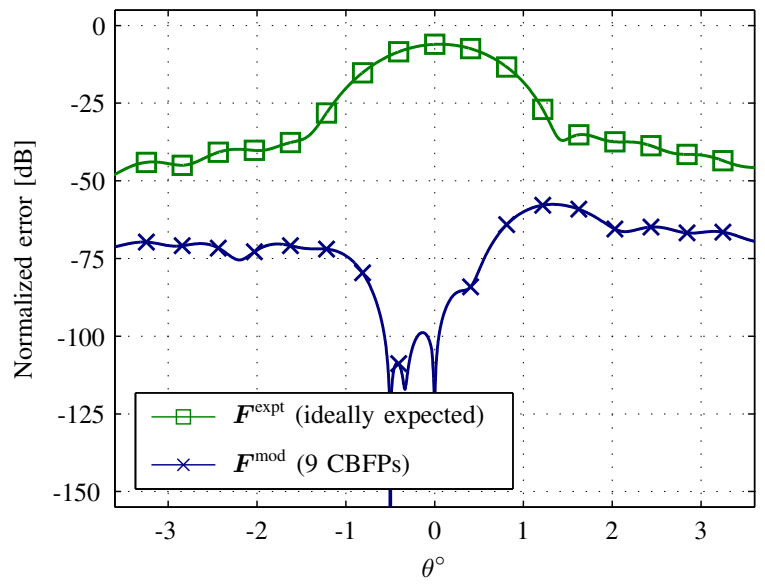

(d) Error function @ $1.75 \mathrm{GHz}\left(\phi=157.5^{\circ}\right)$

Fig. 6. Normalized gain patterns at (a) $580 \mathrm{MHz}$ and (b) $1.75 \mathrm{GHz}$ comparing the reference pattern $\boldsymbol{F}^{\text {ref }}$, modeled pattern $\boldsymbol{F}^{\text {mod }}$, and the expected pattern $\boldsymbol{F}^{\text {expt }}$ for the worst case subreflector displacement $\boldsymbol{\delta}=[7.1,-5.0,18.5] \mathrm{mm}$. Normalized error patterns for the modeled and expected patterns for the same subreflector displacement are also shown in (c) $580 \mathrm{MHz}$ and (d) $1.75 \mathrm{GHz}$. All patterns are shown in the plane of the reference pattern maximum.

The maximum error $\epsilon_{j, N}^{\max }$ is shown in Fig. 5(a) and (b), at the lower and higher frequencies, respectively, and as a function of $N$ for the different sets of CBFPs. The worst case error between the ideally expected and actual reference pattern is shown to range from about $5 \%$ at $580 \mathrm{MHz}$ up to $20 \%$ at $1.75 \mathrm{GHz}$. The importance of improving the conditioning of (4) through the application of the SVD is emphasized by the difference in the results for $S_{1}$ and $S_{2}$. Whereas the worst case error decreases nearly monotonically for $S_{2}$ with every additionally employed CBFP, the reduction in error for using CBFPs from $S_{1}$ is significantly slower. Moreover, the results at $1.75 \mathrm{GHz}$ show that the error in the $S_{1}$ model pattern may even be larger than the error in the expected (uncalibrated) pattern as is evidenced by the error for $N=5$. As before, the use of basis functions from $S_{3}$ is seen to improve the error in the model pattern by approximately a factor five at the lower and a factor two at the higher frequency, respectively, for $N=9$.

Fig. 6(a) and (b) show the reference and CBFP-modeled patterns for the geometrical error resulting in the largest maximum error in the ideally expected pattern at each of the two frequencies. The modeled patterns are produced using CBFPs from set $S_{2}$, with $N=7$ at the lower, and $N=9$ at the upper frequency. For comparison, the ideally expected patterns (no displacement error) at each of the frequencies are also shown, and the actual reference beam pattern at the lower frequency is seen to suffer mainly from a small pointing error, whereas at the higher frequency this error is substantially larger and the sidelobes are also affected significantly. By employing seven CBFPs from $S_{2}$, the lower frequency modeled pattern is visually indistinguishable from the reference pattern, and at the higher frequency the difference between these two patterns is only visible from around the $-30 \mathrm{~dB}$ level. The beam error functions (normalized to the maximum of $\boldsymbol{F}^{\mathrm{ref}}$ ) corresponding to these modeled patterns are shown in Fig. 6(c) and (d), along with the beam error functions (also normalized) for the ideally expected patterns. These error patterns show that the model error is reduced to an extremely low level in the main beam area (over the angular region $\theta<\theta_{\max }$ ), and even over the sidelobe region the error in the model pattern is still well below $-40 \mathrm{~dB}(1 \%)$.

Finally, the effect of $\theta_{\max }$ on the error in $\boldsymbol{F}^{\text {mod }}$ was examined for the worst case geometrical error at $1.75 \mathrm{GHz}$ by increasing the angular region over which the CBFPs are orthonormalized from $\theta_{\max }=0.5^{\circ}\left(\approx 5 \mathrm{~dB}\right.$ level of $\left.\boldsymbol{F}^{\text {expt }}\right)$ to $\theta_{\max }=1.0^{\circ}(\approx$ 
Normalized error $[\mathrm{dB}]$

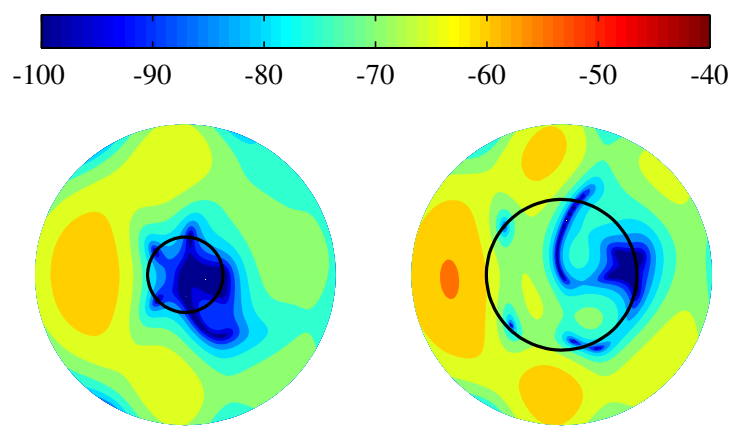

Fig. 7. Normalized error patterns for worst case geometrical error at $1.75 \mathrm{GHz}$ shown over an angular region $\theta \leq 2^{\circ}$. Comparison between (left) $\theta_{\max }=0.5^{\circ}$ and (right) $\theta_{\max }=1.0^{\circ}$.

$20 \mathrm{~dB}$ level of $\boldsymbol{F}^{\text {expt }}$ ). The normalized error patterns resulting for these two cases are shown in Fig. 7, where increasing the angular region is seen to reduce the error in an average sense over a similarly larger region at the price of an increase in error inside the region $\theta<\theta_{\max }$. The testing points for best conditioning of (4) in either case were mostly arranged on or near $\theta=\theta_{\max }$, as can be seen from the deep nulls in the error function where point matching is performed.

\section{CONCLUSIONS AND RECOMMENDATIONS}

The extent to which the sensitivity offered by future radio telescopes can be utilized is dependent on the accuracy with which the antenna beam pattern can be estimated using the least number of calibration measurements. In this contribution a beam estimation procedure based on the Characteristic Basis Function Pattern method [10] is proposed to address this requirement for single beam antennas, and provides a radiation pattern model that can be used to accurately account for instrumental direction-dependent effects during calibration and imaging [6]. Specifically, the method is demonstrated to compensate for certain mechanical deformations that are to be expected in an offset Gregorian antenna, in which case presented simulation results show a reduction in the maximum beam model error of a non-calibrated system from $15 \%$ down to a value less than $0.2 \%$. This level of accuracy is achieved by employing only nine CBFPs to model the actual perturbed beam pattern, and therefore requires as little as nine calibration measurements, indicating that the estimation method is also time-efficient.

The application of the CBFP method to compensate for various other types of system errors, the polarization ambiguity that arises when calibrating on unpolarized sources, and experimental demonstration on an actual antenna system using measured CBFPs, are subjects of ongoing research.

\section{ACKNOWLEDGMENT}

The authors acknowledge O. Iupikov for the development of the GRASP toolbox used in this work.

\section{REFERENCES}

[1] J. Baars, "The measurement of large antennas with cosmic radio sources," IEEE Trans. Antennas Propag., vol. 21, no. 4, pp. 461-474, Jul. 1973.

[2] H. E. Green, "Antenna pattern measurement with a geostationary satellite," Journal of Electrical and Electronics Engineering, vol. 3, pp. 8-17, Mar. 1983.

[3] Y. A. Hafez et al., "Radio source calibration for the Very Small Array and other cosmic microwave background instruments at around 30 GHz," Monthly Notices of the Royal Astronomical Society, vol. 388, no. 4, pp. 1775-1786, Aug. 2008. [Online]. Available: http://dx.doi.org/10.1111/j.1365-2966.2008.13515.x

[4] J. M. Uson and W. D. Cotton, "Beam squint and Stokes V with off-axis feeds," A\&A, vol. 486, no. 2, pp. 647-654, 2008.

[5] Harp, G. R. et al., "Primary Beam and Dish Surface Characterization at the Allen Telescope Array by Radio Holography," IEEE Trans. Antennas Propag., vol. 59, no. 6, pp. 2004-2021, Jun. 2011.

[6] O. M. Smirnov, "Revisiting the radio interferometer measurement equation. II. Calibration and direction-dependent effects," Astronomy \& Astrophysics, vol. 527, p. A107, 2011.

[7] J. E. Noordam, "Measuring Station Beamshapes as a function of time and frequency and in full polarization," in 3GC-II Workshop, Albufeira (Portugal), Sep. 2011.

[8] F. Jensen, P. H. Nielsen, J. Tauber, and A. Martin-Polegre, "Improved In-Flight Pattern Retrieval by Reflector-Deformation Fitting," IEEE Antennas Propag. Mag., vol. 53, no. 5, pp. 215-221, Oct. 2011.

[9] Craeye, C. et al., "Main beam representation in non-regular arrays," in 3GC-II Workshop, Albufeira (Portugal), Sep. 2011.

[10] R. Maaskant, M. V. Ivashina, S. J. Wijnholds, and K. F. Warnick, "Efficient Prediction of Array Element Patterns Using Physics-Based Expansions and a Single Far-Field Measurement," IEEE Trans. Antennas Propag., vol. 60, no. 8, pp. 3614-3621, Aug. 2012.

[11] Aperture Array Calibration and Calibratability Meeting. [Online]. Available: http://www.astron.nl/AACal2012

[12] A. Young, M. A. B. Terada, D. I. L. de Villiers, and D. B. Davidson, "Assessment of the Sensitivity of the South African KAT-7 and MeerKAT/SKA Radio Telescope Reflector Antennas," in Proc. Int. Conf. on Electromagn. in Adv. Applicat. (ICEAA), Cape Town, Sep. 2012, pp. 486-489.

[13] R. Maaskant and M. V. Ivashina, "Characteristic Basis Function Patterns - A Novel Expansion Method for the Fast and Accurate Prediction of Antenna Array Beams," in Proc. Int. Conf. on Electromagn. in Adv. Applicat. (ICEAA), Cape Town, Sep. 2012, pp. 796-799.

[14] P. G. Smith, "Measurement of the Complete Far-Field Pattern of Large Antennas by Radio-Star Sources," IEEE Trans. Antennas Propag., vol. 14, no. 1, pp. 6-16, Jan. 1966.

[15] A. Hartsuijker, J. Baars, S. Drenth, and L. Gelato-Volders, "Interferometric measurement at $1415 \mathrm{MHz}$ of radiation pattern of paraboloidal antenna at Dwingeloo radio observatory," IEEE Trans. Antennas Propag., vol. 20, no. 2, pp. 166-176, Mar. 1972.

[16] I. Theron, R. Lehmensiek, and D. I. L. de Villiers, "The design of the MeerKAT dish optics," in Proc. Int. Conf. on Electromagn. in Adv. Applicat. (ICEAA), Cape Town, Sep. 2012, pp. 539-542.

[17] R. Maaskant, "Analysis of Large Antenna Systems," Ph.D. dissertation, Eindhoven University of Technology, Eindhoven, 2010. [Online]. Available: http://alexandria.tue.nl/extra2/201010409.pdf 


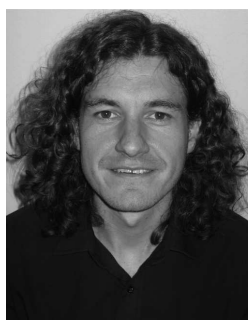

André Young was born in the Free State, South Africa on April 6, 1983. He received the B.Eng. degree in electrical and electronic engineering with computer science (cum laude) and M.Sc.Eng. degree in electronic engineering (cum laude) in 2005 and 2007, respectively, from the University of Stellenbosch, Stellenbosch, South Africa. His master's thesis was on mesh termination schemes for the finite element method in electromagnetics.

He has worked on various engineering projects for Azoteq (Paarl, South Africa) and Entersekt (Stellenbosch, South Africa), and is currently pursuing the Ph.D. degree in electronic engineering at the University of Stellenbosch, where he was also appointed as a Junior Lecturer in 2009 and 2011. Since 2011 he has spent several months as a visiting researcher with the Antenna Group at the Chalmers University of Technology, Gothenburg, Sweden. His main research interests include computational electromagnetics and antennas.

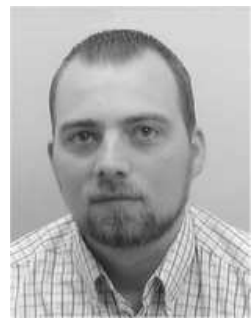

Rob Maaskant (M'11) was born in the Netherlands on April, 14th, 1978. He received his M.Sc. degree (cum laude) in 2003, and his Ph.D. degree (cum laude) in 2010, both in Electrical Engineering from the Eindhoven University of Technology, Eindhoven, The Netherlands. His Ph.D. has been awarded "the best dissertation of the Electrical Engineering Department, 2010." From 2003-2010, he was employed as an antenna research scientist at the Netherlands Institute for Radio Astronomy (ASTRON), Dwingeloo, The Netherlands, and from 2010-2012 as a postdoctoral researcher in the Antenna Group of the Signals and Systems Department at the Chalmers University of Technology, Sweden, for which he won a Rubicon postdoctoral fellowship from the Netherlands Organization for Scientific Research (NWO), 2010. He is currently an Assistant Professor in the same Antenna Group. He is the primary author of the CAESAR software; an advanced integral-equation based solver for the analysis of large antenna array systems. His current research interest is in the field of receiving antennas for low-noise applications, meta-material based waveguides, and computational electromagnetics to solve these types of problems.

Dr. Maaskant received the 2nd best paper prize ('best team contribution') at the 2008 ESA/ESTEC workshop, Noordwijk, and was awarded a Young Researcher grant from the Swedish Research Council (VR), in 2011.

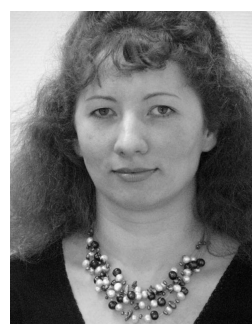

Marianna V. Ivashina (M'11) received a Ph.D. in Electrical Engineering from the Sevastopol National Technical University (SNTU), Ukraine, in 2001. From 2001 to 2004 she was a Postdoctoral Researcher and from 2004 till 2010 an Antenna System Scientist at The Netherlands Institute for Radio Astronomy (ASTRON). During this period, she carried out research on an innovative Phased Array Feed (PAF) technology for a new-generation radio telescope, known as the Square Kilometer Array (SKA). The results of these early projects have led to the definition of APERTIF - a PAF system that is being developed at ASTRON to replace the current horn feeds in the Westerbork Synthesis Radio Telescope (WSRT). Dr. Ivashina was involved in the development of APERTIF during 2008-2010 and acted as an external reviewer at the Preliminary Design Review of the Australian SKA Pathfinder (ASKAP) in 2009. She was a Guest Editor of the special issue of the IEEE Transactions on Antennas and Propagation on Antennas for the Next Generation Radio Telescopes (June, 2011); and since 2011 she assists the SKA office in the development of the statement of work of the next phase of the SKA project. Dr. Ivashinas received several scientific distinctions including the 2nd Best Paper Award ('Best team contribution') at the ESA Antenna Workshop (2008) and the International Qualification Fellowship of the VINNOVA - Marie Curie Actions Program (2009). She is currently Associate Professor at the Antenna Group of the Signals and Systems Department (Chalmers University of Technology, Sweden). Her interests are phased arrays and reflector antennas, antenna system modeling techniques, array signal processing and radio astronomy.

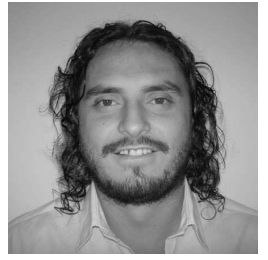

Dirk I.L. de Villiers (S'05-M'08) was born in Langebaan, South Africa, on October 13, 1982. He received the B.Eng and Ph.D. degrees in electrical and electronic engineering from the University of Stellenbosch, Stellenbosch, South Africa in 2004 and 2007 respectively. During 2005 to 2007 he spent several months as visiting researcher with the Computational Modeling and Programming group at the University of Antwerp in Antwerp, Belgium.

From 2008 to 2009 he was a post-doctoral fellow at the University of Stellenbosch working on antenna feeds for the South African SKA program. During this time he was also a part time lecturer at the Cape Peninsula University of Technology. He is currently a senior lecturer at the University of Stellenbosch, and his main research interests include reflector antennas as well as the design of wide band microwave components such as combiners, filters, and antennas.

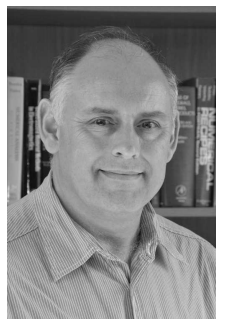

David Davidson David Bruce Davidson (M'85S'07-F'12) was born in London, U.K., 1961. He was raised and educated in South Africa, receiving the B.Eng, B.Eng (Hons), and M.Eng degrees (all cum laude) from the University of Pretoria, South Africa, in 1982, 1983, and 1986 respectively, and the $\mathrm{Ph} . \mathrm{D}$. degree from the University of Stellenbosch, Stellenbosch, South Africa, in 1991.

Following national service (1984-5) in the then South African Defence Force, he was with the Council for Scientific and Industrial Research, Pretoria, South Africa, prior to joining the University of Stellenbosch in 1988. As of 2011, he holds the South African Research Chair in Electromagnetic Systems and EMI Mitigation for SKA there. He was a Visiting Scholar at the University of Arizona in 1993, a Visiting Fellow Commoner at Trinity College, Cambridge University, England in 1997, a Guest Professor at the IRCTR, Delft University of Technology, The Netherlands, in 2003, and an Honorary Visitor, University of Manchester, UK in 2009. His main research interest through most of his career has been computational electromagnetics (CEM), and he has published extensively on this topic. He is the author of the recently revised text "Computational Electromagnetics for RF and Microwave Engineering" (Cambridge, U.K.: Cambridge Univ. Press, 1st edn, 2005, 2nd end, 2011). Recently, his interests have expanded to include engineering electromagnetics for radio astronomy.

Prof. Davidson is a member of the South African Institute of Electrical Engineers and the Applied Computational Electromagnetic Society. He is a recipient of the South African FRD (now NRF) President's Award; presently, he has a B2 research rating from the NRF. He received the Rector's Award for Excellent Research from the University of Stellenbosch in 2005. He is a Past Chairman of the IEEE AP/MTT Chapter of South Africa, and served as an Associate Editor of the IEEE Antennas And Wireless Propagation Letters ('06-08). Currently, he is the editor of the "EM Programmer's Notebook" column of the IEEE Antennas And Propagation Magazine, and is serving on the IEEE Antennas and Propagation AdCom ('11-'13). He served as General Chair of the "8th International Workshop on Finite Elements for Microwave Engineering", held in Stellenbosch, May 2006. He was Chair of the local organizing committee of ICEAA-IEEE APWC-EEIS'12, held in Cape Town in September 2012. 